\title{
Frequency and origin of triploidy in the fire ant Solenopsis invicta
}

\author{
MICHAEL J . B. KRIEGER*†, KENNETH G. ROSS $\ddagger$, CHRISTINA W. Y. CHANG $\dagger$ \\ \& LAURENT KELLER† \\ $\dagger$ Institute of Ecology, University of Lausanne, BB, 1015 Lausanne, Switzerland, †Department of Entomology, \\ University of Georgia, Athens, GA 30602-2603, U.S.A.
}

\begin{abstract}
A large microsatellite survey of fire ants of both social forms (monogyne and polygyne) from both the native and introduced ranges (Argentina and the U.S.A.) revealed surprisingly high levels of triploidy $(12 \%)$ in non-reproductive females from an introduced polygyne population in Georgia, U.S.A. Triploid females were not detected among reproductive (egg-laying) queens from this population, among females from monogyne populations in the introduced range or among females of either social form from the native range. The results of complementary analyses with allozyme markers were highly concordant with the individual microsatellite ploidy designations and confirmed the general patterns in the distribution of triploidy observed with microsatellites. Triploidy in the Georgia polygyne population is hypothesized to be linked to the high frequency of diploid males in this population; although most such males are sterile, $2.4 \%$ were found to possess functional reproductive tracts and presumably produce diploid sperm. Mating of such males with polygyne queens is expected to give rise to triploid females at levels close to those observed. Diploid males are absent in monogyne populations and uncommon in the introduced range, thus explaining the lack of triploid females in samples from these sources. The absence of triploid reproductive queens in the Georgia polygyne population may result from subviability of triploid queens or execution of such queens by workers. Our results suggest that triploid females of social Hymenoptera may be more common than appreciated and are most likely to be found in populations with substantial numbers of fertile diploid males.
\end{abstract}

Keywords: allozymes, diploid males, microsatellites, Solenopsis invicta, triploidy.

\section{Introduction}

The fire ant Solenopsis invicta has received considerable attention as a model for studying the colonyand population-level genetic consequences of variation in social organization. This species is particularly interesting because there are two distinctive social forms: the monogyne (M) form, in which colonies have a single egg-laying queen, and the polygyne (P) form, in which colonies have multiple queens (Vargo \& Fletcher, 1987; Ross \& Keller, 1995a). Recent studies have focused on the evolutionary causes and consequences of these alternative social behaviours and have specifically sought to identify the evolutionary importance of gene flow within and between the forms, of the different mating systems and modes of colony founding of the

*Correspondence. E-mail: michael.krieger@izea.unil.ch. forms, and of the striking differences in reproductive success among potential reproductives (Ross \& Keller, 1995a; Ross, 1997). Any genetic information bearing on these topics can enrich our understanding of how social behaviour interacts with the fundamental forces of evolution to fashion genetic variation in wild populations and set the stage for subsequent evolution.

Extensive allozyme studies of a single introduced $\mathbf{P}$ population in northern Georgia, U.S.A., have revealed that some heterozygous workers have asymmetric band intensities at the two electrophoretic staining bands of monomeric enzymes (K. G. Ross, personal observation). This pattern might be explained if these individuals are triploids (Dybdahl \& Lively, 1995). Indeed, studies of single-cell nuclear DNA amounts using flow cytometry have also suggested the presence of triploids in the same population(J.S. Johnston \& S. B. Vinson, unpubl.obs.). 
This possibility cannot be tested directly using the allozymes, however, because none of the polymorphic allozyme loci described has more than two common alleles in U.S.A. populations (Shoemaker et al., 1992). On the other hand, the recent development of polymorphic microsatellite markers in these ants provides a new tool for investigating triploidy, because each of the six loci that has been developed has more than two alleles (Krieger \& Keller, 1997).

The first aim of this study was to use microsatellites to establish whether or not triploid females occur in the $\mathbf{P}$ population of $S$. invicta in Georgia. The second aim was to identify the possible cause of triploidy if such individuals were found. The most likely explanation is that diploid males in the $\mathbf{P}$ population occasionally produce viable, unreduced diploid sperm and that such males mate with $\mathbf{P}$ queens, producing normal haploid eggs. Although social Hymenoptera are characterized by a male haploid genetic system, diploid males can be produced in some circumstances. Sex is determined by heterozygosity at one or a few loci, with heterozygous individuals becoming females and either hemizygous (haploid) or homozygous (diploid) individuals becoming males (Crozier, 1977). The production of diploid males thus depends on the sex locus genotypes of queens and their mates, with diploid males being produced by queens that mate with a male carrying a sex allele identical to one of the queen's alleles (Crozier, 1977).

In contrast to other species of Hymenoptera (Cook \& Crozier, 1995), diploid males are common in $\mathbf{P}$ populations of $S$. invicta in the United States, representing $73-100 \%$ of all males produced (Ross \& Fletcher, 1985; Ross et al., 1993). This high frequency of diploid males stems from a loss of genetic diversity at the sex-determining locus that occurred when $S$. invicta was introduced into the U.S.A. (Ross et al., 1993). In the native range, where overall genetic diversity is much higher than in the U.S.A., the frequency of diploid males is relatively low, with the proportion of such males being less than $20 \%$ in two $\mathbf{P}$ populations studied in Argentina. Although common in $\mathbf{P}$ nests in the U.S.A., diploid males are absent from mature $\mathbf{M}$ nests. This difference results from the differential mortality of nests producing such males in the two forms. Colonies founded independently by $\mathbf{M}$ queens that produce diploid males invariably die early in development, because resources are diverted from the production of workers (the labour of which is crucial to early colony growth and survival) to the production of males (Ross \& Fletcher, 1985). This source of mortality does not afflict the $\mathbf{P}$ form, because colo- nies are usually founded by budding, during which a sizeable worker force is already present to ensure sustained colony growth and survival.

Two testable predictions stem from the hypothesis that diploid males are responsible for the occurrence of triploidy in some $S$. invicta females. First, triploids should be restricted to $\mathbf{P}$ nests, because $\mathbf{M}$ queens invariably mate with $\mathbf{M}$ males (Shoemaker \& Ross, 1996), all of which are haploid. Secondly, the frequency of triploids should be much higher in introduced than in native $\mathbf{P}$ populations, because of the higher prevalence of diploid males in introduced populations.

Another important factor to be considered in the above scenario is the relative fertility and mating success of diploid and haploid males. It is generally assumed that diploid males are always infertile (aspermic) in S. invicta, as they often are in other social Hymenoptera in which they survive to maturity (Whiting, 1961; Smith \& Wallace, 1971). Indeed, morphological studies have shown that the reproductive system of a high proportion of $\mathbf{P}$ males does not mature properly. Instead of the testicular lobes progressively decreasing in size while spermatozoa are transferred to the vas deferens, the reproductive tract of such adult males remains at a stage of development similar to that of pupae. Chromosome studies revealed that males failing to undergo testicular lobe development are all diploid (Hung et al., 1974). However, insufficient individuals were analysed to determine whether diploid males are invariably sterile. Therefore, the third aim of this study was to investigate whether some diploid males have a normal reproductive system and thus are competent to produce viable (presumably diploid) sperm.

\section{Materials and methods}

\section{Sample collection}

Specimens of S. invicta were collected from the native range in Argentina and the introduced range in the U.S.A. (Table 1). The social form of each sampled nest was determined as described in Ross et al. (1997). The samples from Argentina originated from two localities in the north of the country. One of these is in Corrientes province just south of the city of Corrientes, and the other is in Formosa province and includes the city of Formosa as its centre. Both social forms occur sympatrically in both localities. A total of $36 \mathbf{M}$ and $43 \mathbf{P}$ nests from Corrientes and $35 \mathbf{M}$ and $35 \mathbf{P}$ nests from Formosa were sampled. Collected individuals were workers or 
Table 1 Samples of fire ants collected for determination of ploidy level

\begin{tabular}{lrlllll}
\hline $\begin{array}{l}\text { Class of } \\
\text { individual }\end{array}$ & $\begin{array}{c}\text { Sample } \\
\text { size }\end{array}$ & Country & Locality & $\begin{array}{c}\text { Social } \\
\text { form }\end{array}$ & $\begin{array}{c}\text { Collection } \\
\text { context }\end{array}$ & $\begin{array}{c}\text { Genetic } \\
\text { markers used }\end{array}$ \\
\hline Workers or non-reproductive queens & 36 & Argentina & Corrientes & M & One per nest & MS \\
Workers or non-reproductive queens & 43 & Argentina & Corrientes & P & One per nest & MS \\
Workers or non-reproductive queens & 35 & Argentina & Formosa & M & One per nest & MS \\
Workers or non-reproductive queens & 35 & Argentina & Formosa & P & One per nest & MS \\
Non-reproductive queens & 22 & U.S.A. & Florida & M & On ground after mating flight MS \\
Non-reproductive queens & 42 & U.S.A. & Georgia & M & On ground after mating flight MS \\
Non-reproductive queens & 36 & U.S.A. & Georgia & M & One per nest & MS \\
Egg-laying queens & 65 & U.S.A. & Georgia & $\mathbf{P}$ & One per nest & MS \\
Non-reproductive queens & 388 & U.S.A. & Georgia & $\mathbf{P}$ & 15-20 per nest & A \\
Non-reproductive queens & 90 & U.S.A. & Georgia & $\mathbf{P}$ & One per nest & MS, A \\
Small workers & 41 & U.S.A. & Georgia & $\mathbf{P}$ & One per nest & MS, A \\
Large workers & 103 & U.S.A. & Georgia & $\mathbf{P}$ & One per nest & A \\
Males & 150 & U.S.A. & Georgia & $\mathbf{P}$ & 8-46 per nest &
\end{tabular}

M, monogyne social form; P, polygyne social form; MS, microsatellites; A, allozymes.

non-reproductive queens (young winged queens that had not yet departed on a mating flight). The samples from the U.S.A. were collected from Florida and Georgia. The samples from Florida consisted of 22 non-reproductive $\mathbf{M}$ queens collected immediately after a mating flight. The $\mathbf{M}$ samples from Georgia included 42 non-reproductive queens collected after a mating flight and an additional 36 non-reproductive queens collected directly from nests. The $\mathbf{P}$ sample from Georgia consisted of 65 egg-laying queens, 90 non-reproductive queens, 103 large workers and 41 small workers. For the samples collected from nests, only one individual per nest was used for the genetic analyses reported here. The queens collected after mating flights can be assumed to originate from different nests, because young queens depart synchronously from many nests in a large area to form huge mating swarms. An additional 388 non-reproductive $\mathbf{P}$ queens (from 20 nests) and $150 \mathbf{P}$ males (from nine nests) were collected from the tops of their nests as they were preparing to depart on mating flights.

\section{Genetic data}

All individuals except the males and the 388 non-reproductive queens collected from the tops of $\mathbf{P}$ nests in Georgia were genotyped using six microsatellite loci (Sol-6, Sol-11, Sol-20, Sol-42, Sol-49, Sol-55). These loci possess 17, 15, 17, 26, 18 and 16 alleles, respectively, in native populations and 3, 7, 8, 9, 7 and 8 alleles in introduced populations. Primer sequences and detailed methods are described in
Krieger \& Keller (1997). Individuals possessing three distinct alleles were scored as triploids.

All non-reproductive queens and large workers from the $\mathbf{P}$ nests in Georgia were genotyped with allozymes. Small workers could not be genotyped because they did not contain enough material for both allozyme and microsatellite analyses. Allozyme genotypes at three monomeric allozyme loci $(G p-9$, Pgm-1, Pgm-3) were determined by means of starch gel electrophoresis followed by specific histochemical staining or non-specific protein staining (methods in Shoemaker et al., 1992). The 388 non-reproductive queens were genotyped using only the two most informative allozyme loci ( $G p-9$, Pgm-3). Ploidy levels were estimated from these allozymes by identifying individuals with two-banded staining patterns that showed uneven band intensities (Dybdahl \& Lively, 1995). Gp-9 is a particularly useful marker for detecting triploidy in polygyne $S$. invicta because of the very high level of individual heterozygosity at this locus (Ross, 1997). Heads and thoraces were used for the allozyme analyses, whereas the gasters were used to extract DNA for the microsatellite genotyping. Samples for which both allozyme and microsatellite genotypes were obtained were genotyped blindly, that is without knowledge of the ploidy designations obtained from the other class of markers.

To determine the ploidy level of males, they were weighed and their heads and thoraces were used to obtain their genotypes at five allozyme loci (Aat-2, G3pdh-1, Est-4, Pgm-1, Pgm-3; see Shoemaker et al., 1992). Samples with a single electrophoretic band at 
all loci were provisionally scored as haploids, whereas those showing heterozygous banding patterns at one or more loci were scored as diploids. Ross \& Fletcher (1985) showed that diploid males are heavier and have larger thoraces than haploid males. Accordingly, a distinct bimodal distribution of weights was found for the males surveyed in the present study, with all confirmed diploids weighing more than $7.5 \mathrm{mg}$ (data not presented). Thus, the relatively few males exhibiting single bands at all five loci but weighing more than $8.0 \mathrm{mg}$ were considered to be diploids that were multilocus homozygotes.

\section{Dissection of males}

The abdomens of all males were dissected with fine forceps under a dissecting microscope. The testes and accessory glands were separated and placed in $50 \%$ acetic acid for $1 \mathrm{~min}$ until they became transparent; they were then placed on a slide with a drop of $50 \%$ acetic acid. The slides were covered with a coverslip, pressed by hand to squeeze the organs flat and heated over a flame until the acetic acid evaporated. The coverslips were then carefully removed and the slides put in a $2.5 \%$ Giemsa solution for $15 \mathrm{~min}$. Coverslips were glued in place over the dried specimens to prepare them for observation under the microscope. Characterization of male reproductive tracts was undertaken without knowledge of the ploidy level of these individuals as inferred from the allozymes and weights.

\section{Results}

\section{Triploid females: microsatellite data}

Among the 548 individuals from the four different localities that were genotyped, triploid females were detected only in the $\mathbf{P}$ sample from Georgia. In none of the four samples of the two social forms from Argentina nor in the two $\mathbf{M}$ samples from the U.S.A. were triploids found.

Overall, $7.7 \%$ of the 299 females screened from the $\mathbf{P}$ nests in Georgia possessed three different alleles at one or more loci. The proportions of detected triploids in the different classes of females, $13.6 \%$ in large workers, $9.8 \%$ in small workers, $5.6 \%$ in non-reproductive queens and $0 \%$ in egg-laying queens, differ significantly from one another (contingency test with Williams correction, $G_{\text {adj }}=14.835$, d.f. $=3, P=0.002$ ). Three lines of evidence suggest that the complete absence of triploids among functional queens is not an artifact. First, the proportion of detected triploids was significantly lower among functional queens than among all of the individuals in the other classes combined (contingency test with Williams correction, $G_{\text {adj }}=11.396$, d.f. $=1$, $P<0.001)$. Secondly, the proportion of triploids did not vary significantly among the classes once functional queens were excluded from the analysis (contingency test with Williams correction, $G_{\text {adj }}=3.531$, d.f. $=2, P=0.171$ ), the overall proportion of triploids in this case being 9.8\%. Finally, allozyme surveys have revealed no sign of triploid, egg-laying queens in the Georgia $\mathbf{P}$ population, even though very large numbers of such queens have been genotyped (see below). Although the frequency of triploidy does not differ significantly between large and small workers (contingency test with Williams correction, $G_{\text {adj }}=0.397, \quad$ d.f. $=1$, $P=0.529$ ), the trend towards a higher proportion of large workers that are triploid is consistent with the results of another study reporting a positive association between worker weight and ploidy in this population (M. Goodisman, P. Mack, D. Pearse \& K. Ross, unpubl. data).

The chance of a triploid having three distinct alleles at a given locus, and hence the probability of being correctly identified as a triploid, depends on the effective number of alleles per locus. The actual proportion of triploid females in the Georgia $\mathbf{P}$ population can be estimated from the proportion detected using an iterative method developed by Krieger \& Keller (1998). Functional queens were not considered in this analysis because of the apparent lack of triploids in this class. The proportion of detected triploids and the estimated actual proportion of triploids is given for each locus separately in Fig. 1. The estimated proportion differs significantly among loci (contingency test with Williams correction, $G_{\text {adj }}=21.387$, d.f. $=5$, $P<0.001$ ), mainly because of the effect of one locus, Sol-11, which provided an unusually low estimate of the frequency of triploidy. When this locus is excluded, the estimated proportion of triploids does not vary significantly among loci (contingency test with Williams correction, $G_{\text {adj }}=7.958$, d.f. $=4$, $P=0.735)$. We used the multilocus procedure of Krieger \& Keller (1998) to obtain an overall estimate of the proportion of triploids. Because of the presumed unreliability of locus Sol-11 in revealing triploids, we did not consider this locus in the analysis. The proportion of triploids remaining undetected (i.e. not having three distinct alleles at one or more of the five loci) was $2.3 \%$, yielding an overall estimate of $12.2 \%$ triploids among the non-reproductive females genotyped in this population. Inclusion of the locus Sol-11 in the analysis had only a minor effect on the estimated proportion of 


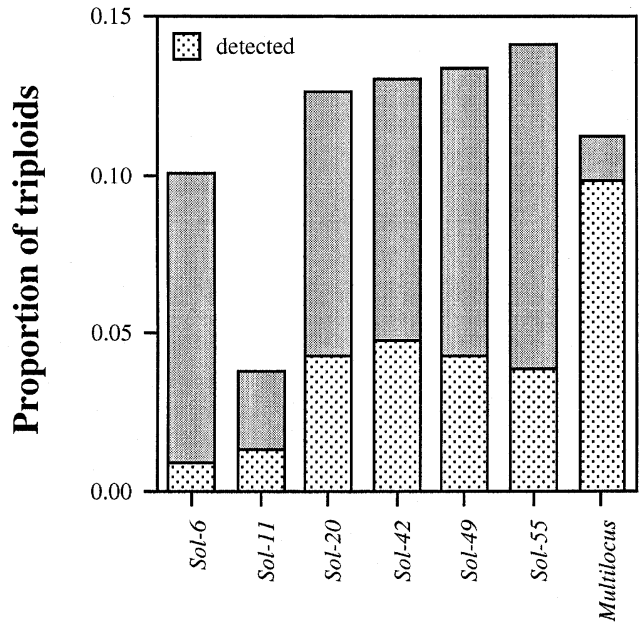

Locus

Fig. 1 Detected and estimated actual proportions of triploid females determined using each of six microsatellite loci in a polygyne fire ant population from Georgia, U.S.A. (functional queens are excluded). The entire bar equals the estimated proportion of triploids. The detected and estimated proportions calculated with a multilocus procedure are given in the last bar.

triploids remaining undetected $(1.3 \%)$ and the overall proportion of triploids $(11.2 \%)$. The estimated proportions of triploids differ little from the proportion detected $(9.8 \%)$, as expected given the relatively large numbers of microsatellite alleles and loci surveyed.

The detected and estimated actual proportions of triploids in each class of females (except reproductive queens) obtained using the multilocus procedure are shown in Fig. 2. Again, the estimated proportion of triploids in each class is only slightly greater than the proportion detected, and the pattern among the classes is the same whether or not the multilocus correction is made: large workers have the highest levels and non-reproductive queens the lowest levels of triploidy.

\section{Comparison of microsatellite and allozyme data}

Eleven of the 193 females genotyped with both allozymes and microsatellites $(5.7 \%)$ were identified as triploids with the allozymes. All of these individuals were large workers; no triploids were detected among non-reproductive or functional queens in the jointly surveyed sample using allozymes (small workers were not genotyped with these markers). However, triploids were detected in the second sample of non-reproductive queens genotyped only

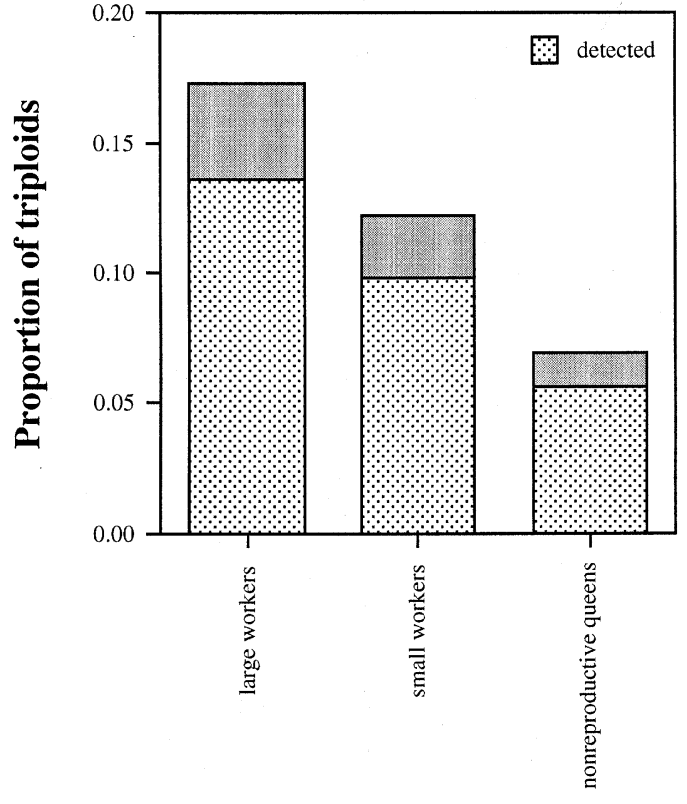

\section{Class of females}

Fig. 2 Detected and estimated actual proportions of triploid females of three different classes determined using five microsatellite loci (locus Sol-11 is excluded) in a polygyne fire ant population from Georgia, U.S.A. The entire bar equals the estimated proportion of triploids.

with allozymes. Thirty-one of the 388 nonreproductive queens in this sample $(7.9 \%)$ were classified as triploids. This second analysis also revealed a significant difference among the 20 source colonies in the proportions of triploid queens (contingency test with Williams correction, $G_{\text {adj }}=66.619$, d.f. $\left.=19, P<0.001\right)$.

There was good agreement between the two types of markers in determining the ploidy level of females. Among the individuals that were analysed using both allozymes and microsatellites, 20 of the 103 large workers and five of the 90 non-reproductive queens had a single band at each of the three surveyed allozyme loci. Because it is impossible to determine the ploidy of such individuals, we excluded them from the comparison of the allozyme and microsatellite data. Of the remaining 168 individuals, 160 (95.2\%) were attributed the same ploidy level (diploid or triploid). Seventy-nine out of 83 large workers $(96.3 \%)$ and 81 out of 85 non-reproductive queens $(95.3 \%)$ were scored identically using the two types of markers. Seven of the individuals with incongruent scores were designated as diploids with the allozymes but were found to be triploids with the microsatellites. One individual was 
scored as a triploid with the allozymes but as a diploid with the microsatellites. Given that $1.3 \%$ of the triploids are expected not to have three distinct alleles at any of the six loci, this individual might well have been a triploid.

\section{Ploidy and sperm production of polygyne males}

Determination of the ploidy of the $150 \mathbf{P}$ males by means of their allozyme banding patterns and weights revealed that $25(16.7 \%)$ were haploid and $125(83.3 \%)$ were diploid. Dissection showed that 28 of the males $(18.7 \%)$ had a complete reproductive system. Among the remaining males, 121 (80.7\%) lacked testes, and one male $(0.6 \%)$ lacked the entire reproductive tract. Slides made from the testes and vasa deferentia revealed that all males with a complete reproductive tract also had sperm, and all males with an incomplete or missing tract were aspermic. All of the haploid males produced sperm and were thus considered fertile (Fig. 3). Significantly, although most of the 124 diploid males were aspermic, three of them (2.4\%) had complete reproductive tracts and produced sperm.

\section{Discussion}

A substantial proportion of small workers, large workers and non-reproductive queens are triploids in the polygyne (P) population of introduced $S$. invicta from Georgia that we studied. Overall, 9.8\%

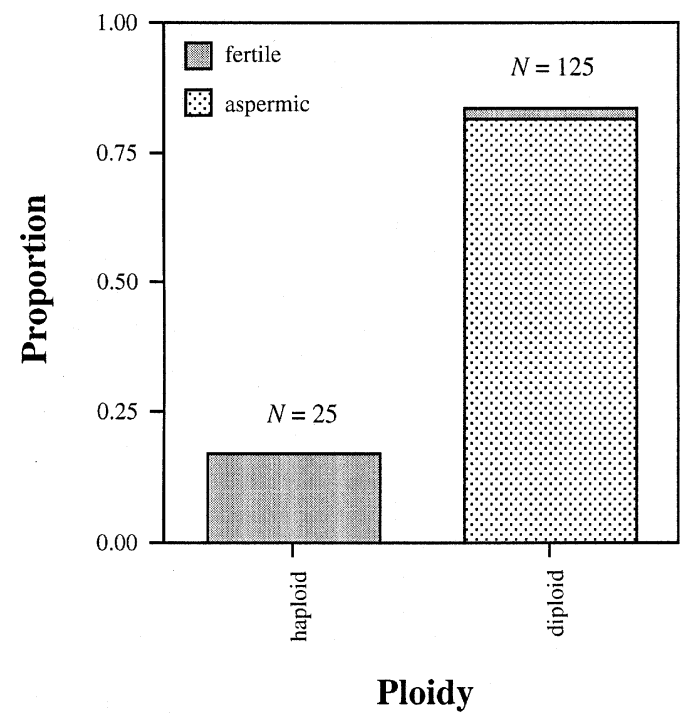

Fig. 3 Proportion of fertile and aspermic haploid and diploid males in a polygyne fire ant population from Georgia, U.S.A. of these individuals carried three distinct alleles at one or more microsatellite loci, and the actual proportion of triploids estimated with the multilocus procedure of Krieger \& Keller (1998) was $12.2 \%$ (or $11.2 \%$ when locus Sol-11 was included).

In contrast to the situation in workers and non-reproductive queens, no triploids were detected among reproductive queens from the Georgia $\mathbf{P}$ population. It is unlikely that this result is caused by a sampling effect, as the binomial probability of not detecting triploids among queens if they actually occurred at the same frequency as in other females $(12.2 \%)$ is only 0.004 . Furthermore, a recent allozyme study of 1535 reproductive queens from this same population (Ross, 1997) revealed no evidence of triploidy. The reason for the complete absence of triploids among reproductive queens is unclear. Triploid queens may be subviable and die prematurely from endogenous causes before becoming reproductively active, or they may be destroyed by workers before or as they attempt to become egg-layers. Evidence for the former possibility is that the frequency of triploidy in non-reproductive queens is less than in either large or small workers. Evidence for the latter possibility is that $\mathbf{P}$ workers discriminate among potential new reproductive queens recruited into their nest based on queen genotype at two protein-coding genes (Ross, 1992, 1997; Keller \& Ross, 1993), and workers may discriminate among queens on the basis of other genetic differences as well. Another relevant issue is the occurrence of triploid males. As some of the triploid individuals will be homozygous at the sex-determining locus, one expects to find males and females among the triploid offspring. Indeed, if triploid males are viable, the proportion of males among triploids should be similar to the proportion of males among diploids. However, none were apparent from the allozyme banding phenotypes in the sample of males that we studied.

Three lines of evidence suggest that diploid males that produce viable diploid sperm might be an important or even the sole source of triploid females in the polygyne study population. First, no triploids were detected in the two monogyne $(\mathbf{M})$ populations studied in the U.S.A. This pattern is expected if triploid daughters arise from $\mathbf{P}$ queens that mate with fertile diploid males, because queens from $\mathbf{M}$ populations mate almost exclusively with $\mathbf{M}$ males, and these males are all haploid (Ross \& Fletcher, 1985; Ross \& Keller, 1995a; Shoemaker \& Ross, 1996). Secondly, the two South American localities contained only diploid females. Again, no or only a low proportion of triploids are expected in South 
America because diploid males are much less common there than in introduced populations.

The third line of evidence linking female triploidy to male diploidy is that a small proportion of diploid males $(2.4 \%)$ were found to produce sperm. Although accounting for only a minority of $\mathbf{P}$ males, simple calculations show that fertile diploid males may well be the sole source of triploid workers. In our sample, $83.3 \%$ of males were found to be diploids, a value close to that found in previous studies of the same population (Ross \& Fletcher, 1985; Ross, 1993). Ross \& Keller (1995b) estimated that $40-60 \%$ of the queens at the sites at which our study material was collected mate with $\mathbf{P}$ males. Assuming that fertile diploid males have the same mating success as haploid males, the expected proportion of $\mathbf{P}$ queens mating with fertile diploid males is thus $5.0-7.5 \%$ (this calculation considers only queens that mate with fertile males). This would lead to proportions of triploid females of the same magnitude, assuming that the viability and lifespan of triploid females is similar to that of diploids. These values are reasonably close to the estimated overall proportion of triploids derived from the microsatellite data $(12.2 \%)$. The proportions of diploid males in the two South American $\mathbf{P}$ populations are $9.8 \%$ and $16.4 \%$ for Corrientes and Formosa respectively (Ross et al., 1993). Assuming the same proportion of fertile diploid males $(2.4 \%)$ and the same frequency of intraform mating by $\mathbf{P}$ queens for the South American sample as for Georgia, the expected proportions of triploid females in the Corrientes and Formosa $\mathbf{P}$ populations are only about $0.002 \%$ and $0.004 \%$. Thus, it is not surprising, given our sample sizes, that we did not detect any triploids in South America.

Diploid males have been reported in other species of Hymenoptera, including the ants Formica ( $F$. aquilonia, F. lugubris, $F$. polyctena, $F$. rufa, $F$. truncorum) (Pamilo et al., 1994), Lasius alienus (Pearson, 1983), Leptothorax muscorum (Loiselle et al., 1990) and Rhytidoponera spp. (Ward, 1980), as well as wasps (Strassmann et al., 1994; H. K. Reeve, pers. comm.) and bees (Woyke, 1963; Comargo, 1982; Roubik et al., 1996). Diploid males may be inviable, as in Apis mellifera, where diploid males are cannibalized by workers within hours after eclosion from the egg (Woyke, 1963). Alternatively, diploid males may be viable but infertile. In Neodiprion nigroscutum, diploid males are incapable of inseminating females because of their large size (Smith \& Wallace, 1971) and, in S. invicta, diploid males, although apparently fully viable, are mostly aspermic. Finally, diploid males may be both viable and fertile. In Bombus terrestris, diploid males have smaller testes, produce fewer spermatozoa and die earlier than haploid males, yet some diploid males mate with queens and sire triploid workers (Duchateau \& Marien, 1995). Thus, triploid females may be more commonplace in Hymenoptera than is currently appreciated, and they are most likely to be found in populations with at least moderate frequencies of viable and fertile diploid males.

We found good agreement between the allozyme and microsatellite markers that we used to identify triploids, with $95.8 \%$ of the females assayed with both classes of markers being assigned the same ploidy level. Among the individuals with discordant assignments, most were scored as triploids with microsatellites but as diploids with the allozymes. There are several possible causes of this type of discrepancy. First, ploidy as inferred from the allozymes is based on the somewhat subjective criterion of staining band intensities. Moreover, it is possible that individual triploids differ in their ability to downregulate the expression of their supernumerary genome, which would make some triploids especially difficult to distinguish from diploids using the band intensity criterion. Also, errors may occur in determining the number of bands at microsatellite loci. Polymerase chain reaction (PCR) amplification is sometimes imperfect, resulting in non-allelic DNA products of various sizes (stutter bands). Depending on the location of such stutter bands, diploids might be mistakenly scored as triploids. Although it is impossible to know which source of error is most common, we feel that microsatellites provide a more reliable means of identifying triploids. Finally, one individual was designated as a triploid with the allozymes but as a diploid with the microsatellites. A low proportion of triploid females are expected to be scored as diploids with the microsatellites, because $1.3 \%$ of triploids should not have three different alleles at any of the six microsatellite loci used. Given our sample size for comparing the two types of markers (168), one or two triploids would be expected to be scored inadvertently as diploids with the microsatellites.

Although five out of the six microsatellites provided similar estimates of the actual proportion of triploids, the estimate for the locus Sol-11 was significantly lower than for the other loci. The reason for this discrepancy is unknown. A possible explanation is the presence of null alleles at Sol-11. However, no deficit of heterozygotes was detected at this locus (Markov chain method, $P=0.825$, $\mathrm{SE}=0.007$, calculated with GENEPOP 2.0, Raymond \& Rousset, 1995), as would be expected if such 
alleles were common. Thus, the cause of the low proportion of triploids detected with Sol-11 remains to be determined.

In conclusion, this study reveals a moderate level of triploidy in non-reproductive $S$. invicta females collected from an introduced polygyne population in Georgia. The presence of these triploids is most probably the result of the high frequency of diploid males in this same population, with the common presence of such males attributable to the loss of diversity at the sex-determining locus (loci) in colonizing populations of this ant. It remains to be resolved whether triploid queens can ever become reproductive and produce viable offspring, and also whether the minority of diploid males that are fertile actually produce diploid sperm. If the fertility of diploid males has a genetic basis and if diploid males sometimes produce fertile progeny, then one would predict that genes conferring fertility to diploid males should spread in a population. Future genetic studies of polygyne $S$. invicta populations in the introduced range should provide an answer to this question.

\section{Acknow ledgements}

We thank Andrew Bourke and John Brookfield for comments on the manuscript and Catherine Roger for technical assistance. This work was funded by grants from the Swiss National Science Foundation (grant numbers 31-35584.92, 31-36907.93, 31-40828.94, 31-43330.95 and 31-49679.96), the 'Fonds du 450e', University of Lausanne and the National Geographic Society.

\section{References}

COMARGO, C. A. 1982. Longevity of diploid males, haploid males and workers of the social bee Melipona quadrifasciata. J. Kans. Entomol. Soc., 55, 8-12.

COOK, J. AND CROZIER, R. H. 1995. Sex determination and population biology in the Hymenoptera. Trends Ecol. Evol., 10, 281-286.

CROZIER, R. H. 1977. Evolutionary genetics of the Hymenoptera. Ann. Rev. Ent., 22, 263-288.

Duchateau, M. J. AND MARIEN, J. 1995. Sexual biology of haploid and diploid males in the bumble bee Bombus terrestris. Insect Soc., 42, 255-266.

DYBDAHL, M. F. AND LIVELy, C. M. 1995. Diverse, endemic and polyphyletic clones in mixed populations of a freshwater snail (Potamopyrgus antipodarum). J. Evol. Biol., 8, 385-398.

HUNG, A. C. F., VINSON, S. B. AND SUMMERLiN, J. W. 1974. Male sterility in the red imported fire ant, Solenopsis invicta. Ann. Entomol. Soc. Am., 67, 909-912.
KELleR, L. AND ROSS, K. G. 1993. Phenotypic basis of reproductive success in a social insect: genetic and social determinants. Science, 260, 1107-1110.

KRIEGER, M. J. B. AND KELlER, L. 1997. Polymorphism at dinucleotide microsatellite loci in fire ant Solenopsis invicta populations. Mol. Ecol., 6, 997-999.

KRIEGER, M. J. B. AND KELLER, L. 1998. Estimation of the proportion of triploids in populations with diploid and triploid individuals. J. Hered., 89, 275-279.

LOISElle, R., FRANCOEUR, A., FISCHER, K. AND BUSCHINGER, A. 1990. Variation and taxonomic significance of the chromosome numbers in the neartic species of the genus Leptothorax (Formicidae: Hymenoptera). Caryologia, 43, 321-334.

PAMILO, P., SUNDSTROM, L., FORTELIUS, W. AND ROSENGREN, R. 1994. Diploid males and colony-level selection in Formica ants. Ethol. Ecol. Evol., 6, 221-235.

PEARSON, B. 1983. The classification of morphologically intermediate ants in the Lasius alienus. Insect Soc., 30, $100-105$.

RAYMOND, M. AND ROUSSET, F. 1995. GENEPOP (version 1.2): population genetics software for exact tests and ecumenicism. J. Hered., 86, 248-249.

Ross, K. G. 1992. Strong selection on a gene that influences reproductive competition in a social insect. Nature, 355, 347-349.

Ross, K. G. 1993. The breeding system of the fire ant Solenopsis invicta: effects on colony genetic structure. Am. Nat., 141, 554-576.

ROSS, K. G. 1997. Multilocus evolution in fire ants: effects of selection, gene flow and recombination. Genetics, 145, 961-974.

ROSS, K. G. AND FlETCHER, D. J. C. 1985. Genetic origin of male diploidy in the fire ant, Solenopsis invicta (Hymenoptera: Formicidae), and its evolutionary significance. Evolution, 39, 888-903.

ROSS, K. G. AND KELLER, L. 1995a. Ecology and evolution of social organization: insights from fire ants and other highly eusocial insects. Ann. Rev. Ecol. Syst., 26, 631-656.

ROSS, K. G. AND KELLER, L. 1995b. Joint influence of gene flow and selection on a reproductively important genetic polymorphism in the fire ant Solenopsis invicta. Am. Nat., 146, 325-348.

Ross, K. G., VARGO, E. L., KELler, L. AND TRAGER, J. 1993. Effect of a founder event on variation in the genetic sex-determining system of the fire ant Solenopsis invicta. Genetics, 135, 843-854.

ROSS, K. G., KRIEGER, M. J. B., SHOEMAKER, D. D., VARGO, E. L. AND KELLER, L. 1997. Hierarchical analysis of genetic structure in native fire ant populations: results from three classes of molecular markers. Genetics, 147, 643-655.

ROUBIK, D. W., WeIGT, L. A. AND BONILlA, M. A. 1996. Population genetics, diploid males, and limits to social evolution of euglossine bees. Evolution, 50, 931-935.

SHOEMAKER, D. D. AND ROSS, K. G. 1996. Effects of social organization on gene flow in the fire ant Solenopsis 
invicta. Nature, 383, 613-616.

SHOEMAKeR, D. D., COSTA, J. T. AND ROSS, G. K. 1992. Estimates of heterozygosity in two social insects using a large number of electrophoretic markers. Heredity, 69, 573-582.

SMITH, S. G. AND WALlACE, D. R. 1971. Allelic sex determination in lower Hymenoptera, Neodiprion nigroscutum. Can. J. Genet. Cytol., 13, 617-621.

STRASSMANN, J. E., HUGHES, C. R., TURILlazZI, S., SOlís, C. R. AND QUELLER, D. C. 1994. Genetic relatedness and incipient eusociality in stenogastrine wasps. Anim. Behav., 48, 813-821.
VARGO, E. L. AND FLETCHER, D. J. C. 1987. Effect of queen number on the production of sexuals in natural populations of the fire ant, Solenopsis invicta. Physiol. Entomol., 12, 109-116.

WARD, P. S. 1980 . Genetic variation and population differentiation in the Rhytidoponera impressa group, a species complex of ponerine ants (Hymenoptera: Formicidae). Evolution, 34, 1060-1076.

whiting, A. R. 1961. Genetics of Habrobracon. Adv. Genet., 10, 333-406.

wOYKe, J. 1963. What happens to diploid drone larvae in a honey bee colony. J. Apic. Res., 2, 73-75. 